\title{
Sparse Approach in Filtering of Color Images Corrupted by Mixture Noises
}

\author{
VOLODYMYR PONOMARYOV ${ }^{1 *}$, ALFREDO PALACIOS-ENRIQUEZ $^{1}$ \\ ${ }^{1}$ ESIME-Culhuan, Instituto Politecnico Nacional, 04430, Col. San Fco Culhuacan, \\ Mexico-city, MEXICO
}

\begin{abstract}
A novel filtering approach is presented in denoising in the color images contaminated by mixture of additive-impulsive noises. Novel framework consists of three principal stages: impulsive noise suppression that is performed detecting pixels corrupted by impulsive noise and then, filtering found spikes by a variant of median filter; during second stage, original additive noise suppression filter is employed in Wavelet transform domain via a sparse representation and 3D-filtering; finally, non- desirable effects obtained in an image during previous stages are processed to correct fine details. In case of multiplicative noise suppression, the designed denoising scheme uses 3D homomorphic sparse processing stage and post-filtering procedure. Evaluation of novel approach in denoising complex distortions has been performed using objective criteria (PSNR and SSIM measures) and subjective perception via human visual system confirming their better performance in comparison with state-of-the- art techniques.
\end{abstract}

Keywords: Signal processing, Image Processing, Filtering of Color Images, Mixture Noises

Received: December 2, 2019. Revised: April 11, 2020. Accepted: April 29, 2020. Published: May 16, 2020 .

\section{Introduction}

The fundamental problem in image processing consists in reducing a noise while preserving the most of image features. The presence of random digital noise in an image reduces the performance of different systems such as pattern recognition, diagnostics, object control, etc. Principal difficulties in any filtering technique are that a processing procedure should perform suppression of noise, meanwhile the fine details, edges, and texture properties can be unchanged. The complex and changing structure of real images does not allow the corrupted image details to be efficiently identified and filtered. If fine details of an image are distorted, these drawbacks could cause misinterpretation during medical diagnosis, incorrect classification of objects in the satellite images, erroneous detection of obstacles by autonomous robots, errors in telemedicine applications, etc. [1]. During image acquisition, additive or multiplicative noise can be present, and during its transmission or acquisition, further contamination may be caused by impulsive noise. Images may be corrupted by interference and imperfections in the channel or the reception equipment. Additionally, digital cameras can introduce noise, electronic interference or errors in data acquisition [2]. The most common type of noise additive one is usually assumed to be Gaussian random process $n_{a d}(i, j)$ where all pixels in an image are corrupted. Other type of noise is multiplicative (speckle) one that is usual for coherent sensors such as ultrasound or radar sensors [3].

The most common model of mixed noise used is a combination of additive noise, usually, Gaussian and random impulsive noise $n_{i m}(i, j)$. The corrupted image
$E(i, j)$ in such type of noises can be represented as follows:

$$
E(i, j)= \begin{cases}e(i, j)+n_{a d}(i, j), & \text { probability } 1-\mathrm{p}_{\mathrm{im}} \\ \mathrm{n}_{\mathrm{im}}(\mathrm{i}, \mathrm{j}) & \text {, probability } \mathrm{p}_{\mathrm{im}}\end{cases}
$$

In case of multiplicative noise $\varepsilon(i, j)$, the corrupted image $E(i, j)$ is presented as folllows:

$$
E(i, j)=\varepsilon(i, j) \circ e(i, j)+n_{a d}(i, j)
$$

\section{Related Works}

The restoration of corrupted images by a mixture of different type of noises requires novel approaches, because a lot of existing techniques developed for additive noise suppression are not capable to eliminate the artefacts produced by impulsive noise or other type noise. There are several filtering techniques for Gaussian additive noise elimination where among them, there exist different techniques based on search of a group of pixels called as reference block. Jain [4] proposed a technique based on WT that is applied to some patches with a chosen degree of similarity. Filtering is performed for each sub-band wavelet by obtaining a threshold that adapts to the conditions of each a neighborhood. Lukin [5] proposed an adaptive filter based on an assessment of the image locality via filtering by DCT to obtain a neighborhood and to estimate the local variance, then using it to distinguish homogeneous and heterogeneous areas. Bahoura [6] proposed a signal denoising technique based on wavelet with a thresholding function, which is applied to the wavelet coefficients. Jin [7] introduced new non-local operators to interpret the filter as a regularization of the Dirichlet's functional. Smoothing 
and fidelity are derived from the same geometric principle. Buades [8] proposed a method for measuring noise and comparing performance of the methods in removing image noise. Then, the authors used a new algorithm based on NLM for a nonlocal average of all pixels in an image. Dabov [9] presented the video filtering method VBM3D based on a highly dispersed signal in the local domain of a $3 \mathrm{D}$ transform. This method uses a 3D array called group that is applied to store all blocks similar to the block being processed. The grouping is performed by searching for similar blocks in the space-time domain. For each a 3D group, filtering and shrinkage is performed in the $3 \mathrm{D}$ transform domain. In our previous study [10], we performed similar to BM3D framework (SM3D-DCTNS) using DCT and block matching procedures that demonstrated superior performance in comparison with NML and BM3D techniques. One drawback appears in this kind of filtering is that the found similarity measure may cause the impulses that can be considered as fine details, so the corrupted pixel is not filtered. There are several papers that use ideas of fuzzy logic theory in denoising and can suppress additive or impulsive noise in separated form [11] [12] [13]. For example, in our previous study [12], two frameworks (FMANS 2 and FMANS H) have been designed to suppress additive Gaussian noise but any of these techniques has no ability in filtering complex (impulsive-additive) noise.

Filters applied in denosing for additive noise do not perform a correct restoration of pixels contaminated by impulsive spikes, so it is necessary to filter or to restore such pixels before denosing the pixels that are contaminated by additive noise. There are different techniques for the elimination of impulsive noise, where the detection of noisy pixels or random impulses is performed in the first stage, following these spikes should be suppressed during filtering process. Different techniques for the elimination of impulsive noise are mostly based on use of kind of median filter or their multichannel modification such as Vector Median Filter [14], Switching Median Filter [15], etc. Other techniques are based on the detection of contaminated pixels in the first step, and then, a filtering process should be only applied to corrupted pixels. $\mathrm{Xu}$ [16] proposed an efficient filter for universal impulse noise removal. This method consists of two stages: impulse detection and filtering. For detection, a robust local image statistic, called the extremum compression rank-order absolute difference (ECROAD), is designed to detect impulse noise in an image. For filtering, the universal impulse noise filter is proposed by combining the ECROAD with the NLM. Nasri [17] presented an effective filtering method to remove impulse noise from images. In this two-stage method, the detected noise-free pixels remain unchanged. Then, Gaussian filter with adaptive variances according to the image noise level is applied in denoising stage. Veerakumar [18] introduced an adaptive radial basis function interpolation-based impulse noise removal algorithm. This approach consists of two stages: noisy pixel detection and correction. The radial basis function interpolation scheme is used to estimate the unknown noisy pixel value from the noise-free known neighboring pixel values. For both noisy pixel detection and correction, a center sliding window is considered at each a pixel location.

There are several novel techniques that can remove a mixture of noises, usually additive noise and impulsive noise. Most of these techniques perform the filtering of impulsive noise in a first stage, and the filtering of additive noise is applied during second stage. In [19, 20], suppression techniques for mixed noise (additive Gaussian and impulsive saturated) are proposed. In [19], the impulsive noise detector is based on the differences between a central pixel and its neighbor pixels. When a contaminated pixel is detected, it is replaced by a mean of neighbors. Filtering of additive Gaussian noise is performed using a bilateral filter $(\mathrm{BF})$, in which the $\mathrm{BF}$ parameters are adjusted. Jiang [21] proposed a method to suppress mixed noise called weighted encoding with sparse nonlocal regularization (WESNR). The WESNR algorithm does not use a detector of impulses as an individual stage, so each corrupted block is encoded over a pre-learned dictionary to remove the impulse noise and additive white Gaussian noise simultaneously in a soft impulse pixel detection manner. The suppression of mixed noise is performed by weighting the encoding residual in such a way that the final encoding residual tends to follow a Gaussian distribution. The weighted encoding and sparse nonlocal regularization are unified into a variational framework.

In present study, novel approach in suppressing a additive-impulsive mixture noise and multiplicativeadditive mixture noise is developed. The suppression of mixed additive-impulsive noise is divided in several stages: the suppression of impulsive noise is performed using a detector of impulses and a variant of median filter; the additive noise suppression is performed on wavelet domain that demonstrates better quality, employing the advantage of sparse representation; and, finally, in order to improve the quality of the image, obtained during the previous suppression stages, a postprocessing stage should be applied.

In case of multiplicative-additive mixture noise, the initial stage consists of homomorphic transformation of input image, following sparse framework in denoising, and final postprocessing stage.

\section{Proposed Filtering Approach}

The proposed framework to filter color image corrupted by impulsive-additive noise using Sparse Representation and $3 D$ Wavelet Color Filtering (FMN-3DWT-C) consists of three stages: a) impulse noise detection and filtering, b) additive noise filtering, and c) postprocessing procedure (Fig. 1).

In developed impulsive noise suppression stage, the detection and restoring of pixels contaminated by impulsive noise are performed.

\subsection{Fuzzy Impulsive Noise Detector}

Detection of noisy pixels is a very important stage because a poor detection could generate undesirable 
effects, such as: blurring in areas of fine details or texture distortion.

The detection process in current framework is based on gradient value and fuzzy sets theory. In this stage, all pixels are analyzed using a vicinity of $3 \times 3$ pixels, where several neighbors are assigned to a chosen direction (Northwest (NW), North (N), Northeast (NE), East (E), Southeast (SE), South (S), Southwest (SW), West (W)). The basic gradient value in direction $(k, l)$ of a central pixel in the position $(i, j)$ is defined as follows:

$$
\nabla_{(k, l)} E(i, j)=E(i+k, j+l)-\mathrm{E}(\mathrm{i}, \mathrm{j}) ; k, l \in(-1,0,+1),
$$

where $(k, l)$ belong to one of eight directions. It is necessary to distinguish between corrupted and edge pixels, where two values are defined that are known as basic $\nabla_{R} E(i, j)$ and related gradients $\nabla_{R}^{\prime} E(i, j)$ and $\nabla_{R}^{\prime \prime} E(i, j)$. The related gradients are calculated using neighboring pixels that form a right angle in the same direction as the basic gradient. We use fuzzy logic sets large $(L)$, small $(S)$, big negative $(B N)$ и big positive $(B P)$ in case of the trapezoidal membership functions for $S$ and

$L$ sets:

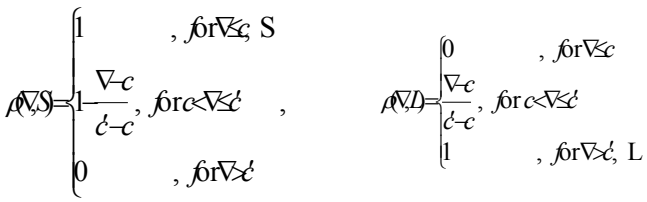

and for $B N$ and $B P$ sets:

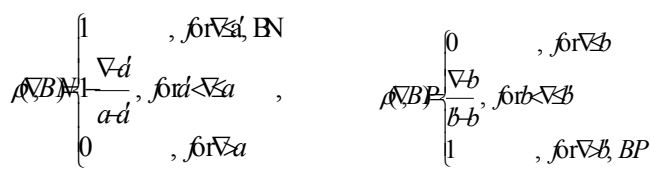

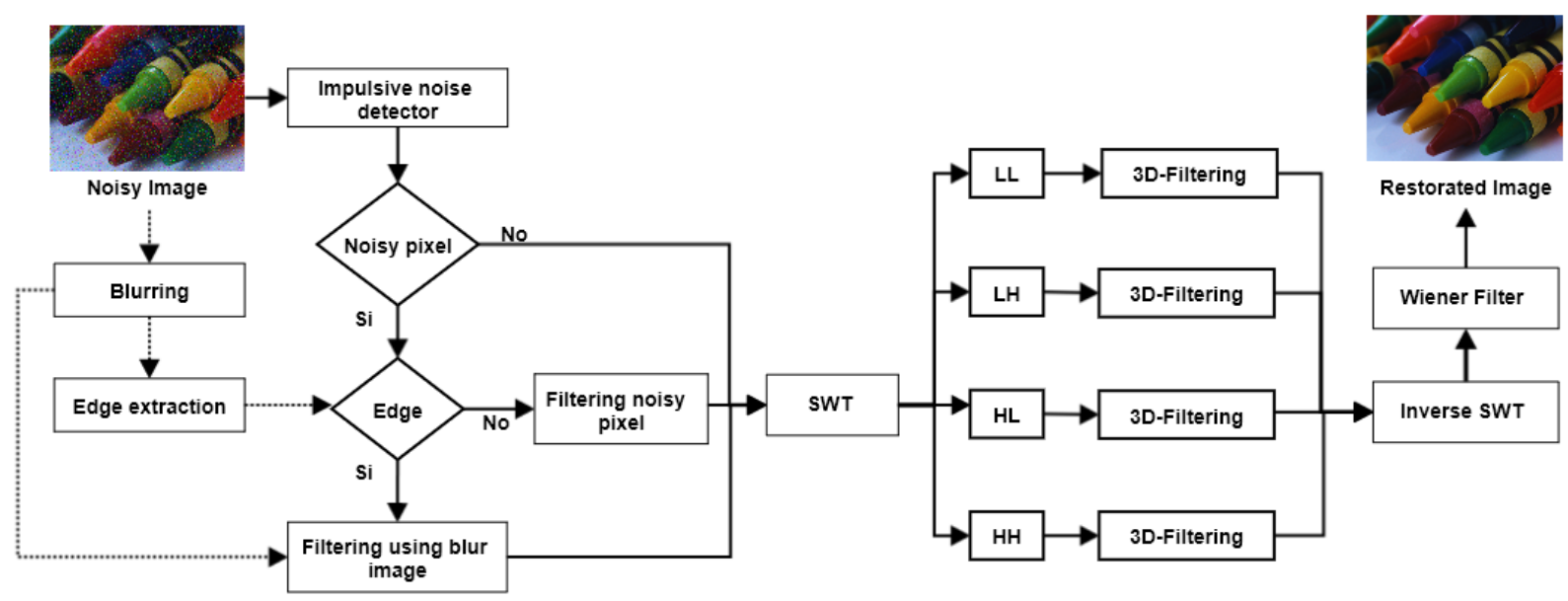

Fig. 1. Block-diagram of proposed method FMN-3DWT-C.

The next step consists of introducing a fuzzy gradient in each a direction to distinguish between a noisy pixel and a noise-free pixel. The fuzzy gradient is defined according to the Fuzzy Rule 1 as follows:

IF $\left[\left|\nabla_{R} A(i, j)\right|\right.$ is $L A N D\left|\nabla_{R}^{\prime} A(i, j)\right|$ is $S$ OR $\left|\nabla_{R} A(i, j)\right|$ is

$L A N D \quad\left|\nabla_{R}^{\prime \prime} A(i, j)\right|$ is $S$ OR $\nabla_{R} A(i, j)$ is $B P \quad A N D$

$\nabla_{R}^{\prime} A(i, j) \quad A N D$ are $B N$ OR $\nabla_{R} A(i, j)$ is $B N A N D$ $\nabla_{R}^{\prime} A(i, j) \quad A N D \quad \nabla_{R}^{\prime \prime} A(i, j)$ are $\left.B P\right]$ THEN Fuzzy Gradient $\nabla_{R}^{F} A(i, j)$ is Large

In order to determine whether a central pixel is contaminated by impulsive noise, the following fuzzy rule is used:

IF most of the eight $\nabla_{R}^{F} A(i, j)$ are $L$ THEN $A(i, j)$ is Noisy.

In particular, if four or more fuzzy gradients are large, then the analyzed pixel is tagged as a noisy pixel. The detection of random spikes is performed on each a pixel. If a pixel is detected as a corrupted one, then their position is tagged, thus generating an image as a map of the corrupted pixels.

\subsection{Restoration of pixels corrupted by impulsive noise}

The restoration of corrupted pixels is performed for each channel RGB in independent form. Let explain below the process for channel $\mathrm{R}$. Once that all impulses in the image are identified and tagged, the next step consists of replacing the noisy pixels using filtering technique. In the filtering of pixels contaminated by impulsive noise, the information generated by the impulsive noise detector is used. Each a pixel is analyzed to know if a noisy pixel exists in its position. In the detection of 
impulses, it cannot be avoided that some pixels that belong to edges and/or textures, are detected as noisy pixels. So, it is necessary to include additional information to correct this fact.

The edge extraction techniques of an image are based in changes of intensity within a neighborhood, so that the impulses could be detected as a edge. In order to extract the edges of an image contaminated by mixed noise, two steps have been proposed:

1) Blurring the image contaminated by mixed noise.

2) Edges extraction.

Firstly, the blurring of noisy image is performed using a median filter with a vicinity of $5 \times 5$ pixels. Finally, the edges extraction is performed using Canny algoritm.

If the position $(i, j)$ is found a tagged as a noisy pixel, following a neighborhood $\mathrm{W}$ of size $3 \times 3$ pixels is taken. Then, the restoration of the pixel is performed considering whether the pixel belongs to an edge or not.

\section{First case. The pixel does not belong to an edge.}

The restoration stage of corrupted pixel is based on the Vector Median Filter (VMF) proposed by Astola [22], and this filter is only applied to those pixels that are marked as noisy ones. Firstly, the sum of absolute differences $(S A D(i . j))$ of each a pixel with its neighbors is performed. The value $\operatorname{SAD}(, j)$ is defined as:

$$
S A D(i, j)=\sum_{k=1}^{3} \sum_{l=1}^{3}|E(i, j)-E(k, l)|
$$

where $(i, j)$ is the position of pixel, and $(k, l)$ are the positions of their neighbors. From this process, nine values are obtained, where the pixel among them in position $(r, s)$ that gives $\min S A D(i, j)$ should be selected as the estimation $E_{\text {no_edge }}(i, j)=E(r, s)$. It should be mentioned that the values tagged as noisy pixels in the neighborhood are not considered in the sum of absolute differences.

\section{Second case. The pixel belongs to an edge.}

When a pixel belongs to an edge, it does not imply that it is not contaminated by impulsive noise, so we obtain a first approximation $E_{1}(i, j)$, considering that the pixel does not belong to an edge. Next, a vicinity $E_{\text {blur }}(i, j)$ from the blur image is taken and a second approximation is obtained as follows: $E_{2}(i, j)=$ median $\left.E_{\text {blur }}(i, j)\right\}$, and finally, the restoration of pixel is defined as :

$\left[E_{1}(i, j)+E_{2}(i, j)\right] / 2$.

\subsection{Additive Noise Filtering}

The additive noise filtering is based on sparse representation and $3 \mathrm{D}$ filtering on WT domain. The techniques that use sparse representation to suppress additive noise are based in the behavior of noise in the domain of chosen transform. Further, the filtering based on shrinkage method allows reducing the additive noise, whereas the edges and fine details suffer less deterioration when such reconstruction is performed [23].
The proposed additive noise filtering stage is performed on WT domain and can be divided in two stages: 1) grouping using block-matching, and 2) 3D-filtering.

The WT produces four sub-bands: LL, LH, HL, and HH. Next, the additive noise suppression is performed to each a sub-band in independent form. The additive noise suppression stage is performed in WT domain where there are applied two processing procedures:

1) Grouping using block-matching, and 2) $3 D$-filtering. The figure 2 shows the process of additive denoising on WT domain.

Grouping via block-matching: The procedure of block matching is performed using the three color channels, the highly similar blocks to a reference block should be located, and these ones are stored in a group. It is necessary to note that the blocks are $2 \mathrm{D}$ arrays and the grouping process [9] is formed as a 3D array.

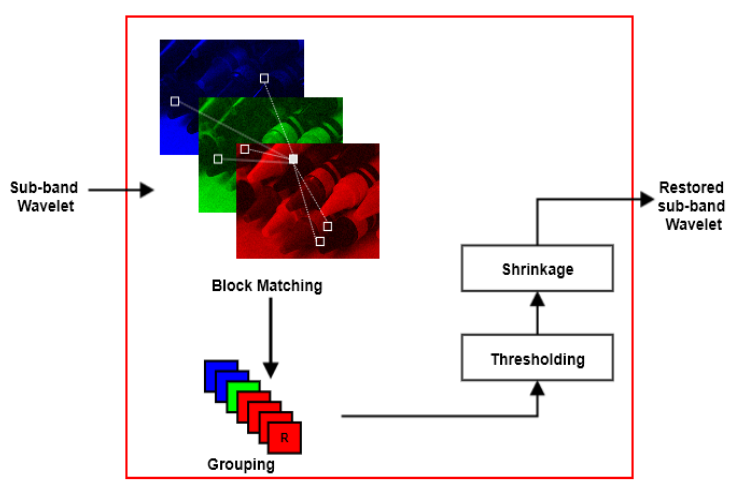

Fig. 2. Filtering process in each sub-band WT.

The similarity degree between two blocks is obtained employing a similarity measure. If the similarity is higher than a chosen threshold, the element of a block can be considered as similar one, and it enters to a current group. In this framework, the sum absolute difference (SAD) is used as matching criterion, which is written as follows:

$$
S A D(p, q)=\sum_{m=1}^{M} \sum_{n=1}^{N} \mid \dot{E}(p, q)-R(p+m . q+n \mid
$$

where $M$ and $N$ are the image dimensions, and $E(p, q)$ is the reference block in the position $(p, q)$. Let denote the reference block as $E(p ; q)$, and then all similar blocks are $E_{r}(p ; q)$.

3D filtering: The designed 3D filtering uses two processes: thresholding and shrinkage. In the thresholding, all wavelet coefficients that belong to each a block of the 3D array are compared with a fixed threshold $\left(T_{W}\right)$. If the absolute value of a coefficient is less than the threshold, this coefficient is replaced with the zero value, as follows:

$$
\hat{E}_{W_{-} 3 D}(p, q, r)=\left\{\begin{array}{c}
E_{W \_3 D}(p, q, r),|| \mathrm{E}_{\mathrm{W}_{-} 3 \mathrm{D}}(p, q, r) \mid \geq T_{W} \\
0, \quad \mathrm{E}_{\mathrm{W}_{-} 3 \mathrm{D}}(\mathrm{p}, \mathrm{q}, \mathrm{r})<\mathrm{T}_{\mathrm{W}}
\end{array}\right.
$$

In the next step, there is performed the shrinkage of the $3 \mathrm{D}$ array, i.e., from the 3D array, there should be obtain an approximation of the $2 \mathrm{D}$ array. This can be performed 
using an averaging filter with chosen weights that depend on similarity measure, as follows:

$$
\begin{gathered}
\hat{E}_{W}(i, j)=\frac{\sum_{l=1}^{k} E_{W \_3 D}(i, j, l) w_{l}}{\sum_{l=1}^{k} w_{l}}, \\
\mathrm{w}_{1}=1-\operatorname{SAD}[\mathrm{E}(\mathrm{i}, \mathrm{j}, \mathrm{l}), \mathrm{E}(\mathrm{i}, \mathrm{j} . \mathrm{k})]
\end{gathered}
$$

Finally, in order to obtain a filtered image $E(i, j)$, the additive noise filtering is applied to each RGB channel of an image.

\subsection{Post-processing}

During previous filtering stages, there are produced some undesirable artefacts, so in the filtered image such artefacts should be corrected. A Wiener filter [24]

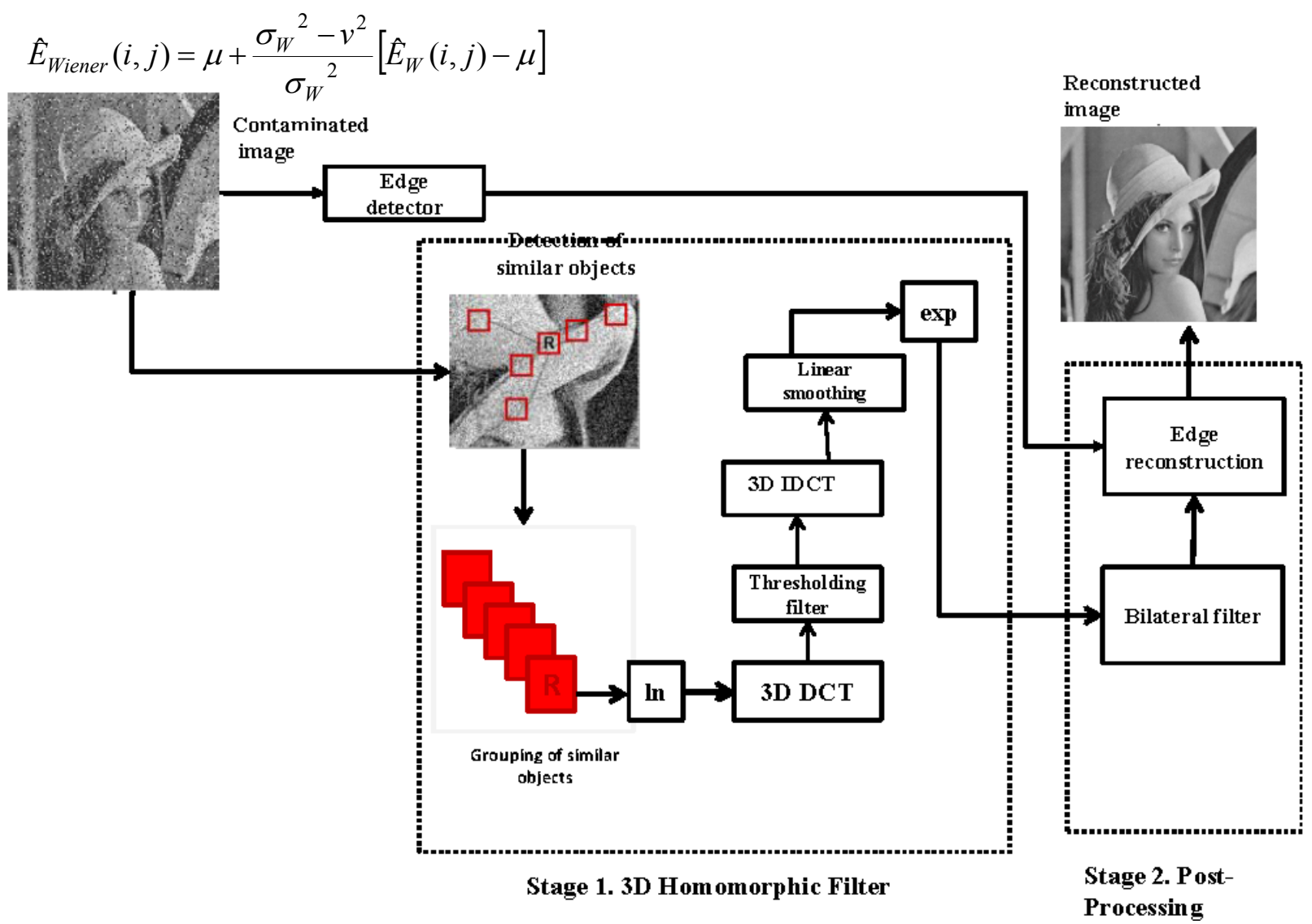

where $\mu$ is local mean, $\sigma_{W}^{2}$ is the local variance, and $v^{2}$ is the average of all the local estimated variances, employed to increase the quality of filtered image.

\subsection{Denoising in case of multiplicative distortion}

In case of multiplicative noise the model of signal degradation defined in form of noise (speckle) and image multiplication [1-3] as presented in (1.1a). So, the proposed framework uses similar spase approach explained in Fig. 3 where designed framework named as Multiplicative Denoising Sparse Representation using DCT (F-MNSR-DCT) consists of two stages: 1) 3D homomorphic sparse filter, and 2) post-processing procedure.

Fig. 3. Block-diagram of proposed technique used for multiplicative denoising via sparse representation (F-MNSR-DCT).

\section{Experimental Results}

The experimental results were performed using a set of 100 color test images proposed by Malinski [25]. Mentioned set contains images with different texture and fine details structure that can guarantee robustness of investigating techniques.

\subsection{Evaluation Criteria}




$$
\operatorname{SSIM}(E, \hat{E})=\frac{\left(2 \mu_{E} \mu_{\overparen{E}}+C_{1}\right)\left(\sigma_{E, \hat{E}}+C_{2}\right)}{\left(\mu_{E}^{2}+\mu_{\hat{E}}^{2}+C_{1}\right)\left(\sigma_{E}^{2}+\sigma_{\hat{E}}^{2}+C_{2}\right)}
$$

In (11), $\mu_{E}$ and $\mu_{\hat{E}}$ determine local means for $E$ and $\hat{E}$, accordingly; $\sigma_{E}^{2}$ and $\sigma^{2} \hat{E}$ are local dispersions for $E$ and $\hat{E}$, and $\sigma_{E, \hat{E}}$ is local cross correlation function for $E$ and $\hat{E}$; constants $C_{n}<<1$

\subsection{Efficiency of the filter FMN-3DWT-C}

The experimental results (PSNR, SSIM) for all test images are shown in Table 1. Additionally, we use the subjective visual perception presenting filtered images and their error images for the best state-of-the-art filters to compare their noise suppression.

Table 1. Average PSNR and SSIM Values

PSNR
\begin{tabular}{|l|l|l|l|l|l|}
\hline $\begin{array}{c}\sigma \\
\%\end{array}$ & 10 & 20 & 30 & 40 & 50 \\
\hline 10 & 34.33 & 33.52 & 31.95 & 28.51 & 24.30 \\
\hline 20 & 29.43 & 28.76 & 27.43 & 25.21 & 22.12 \\
\hline 30 & 25.61 & 25.15 & 24.16 & 22.60 & 20.29 \\
\hline 40 & 23.17 & 22.72 & 21.94 & 20.66 & 18.80 \\
\hline 50 & 21.36 & 20.95 & 20.27 & 19.14 & 17.54 \\
\hline
\end{tabular}

SSIM
\begin{tabular}{|l|l|l|l|l|l|}
\hline $\begin{array}{c}\sigma \\
\%\end{array}$ & 10 & 20 & 30 & 40 & 50 \\
\hline 10 & 0.9873 & 0.9844 & 0.9745 & 0.9365 & 0.8592 \\
\hline 20 & 0.9428 & 0.9343 & 0.9119 & 0.8699 & 0.7958 \\
\hline 30 & 0.8754 & 0.8648 & 0.8416 & 0.8008 & 0.7335 \\
\hline 40 & 0.8192 & 0.8054 & 0.7795 & 0.7394 & 0.6733 \\
\hline 50 & 0.7682 & 0.7535 & 0.7262 & 0.6832 & 0.6204 \\
\hline
\end{tabular}

\subsection{Comparison with state-of-the-art techniques}

There are different techniques for mixed noise suppression. In order to evaluate the proposed method, we compare it with the better existing state-of-the-art techniques: Wiener [24], Bilateral [19], NLM [8] and WESNR [21]. The filter Wiener, Bilateral and NLM were designed to decrease the additive noise only, so it is necessary to perform, previously, the filtering of impulsive noise to compare with our technique.

In figures 4-6, there are shown the visual results obtained to images: pic002, pic029, and pic059 corrupted by impulsive and additive noises with different values of $\%$ and $\sigma$, respectively.
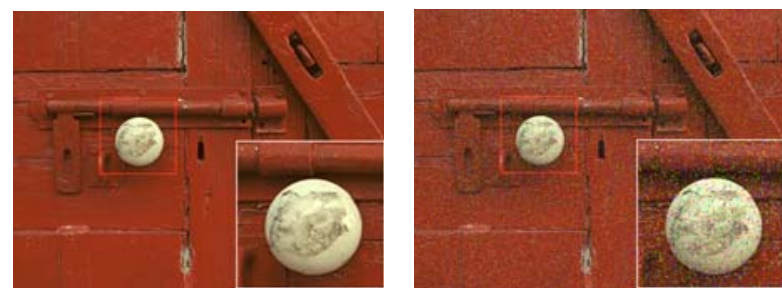

Original

b) Noisy
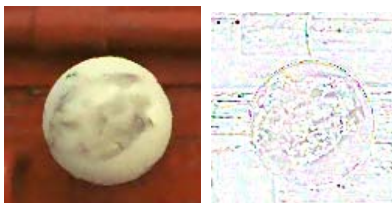

c) Bilateral. d) Bilateral error

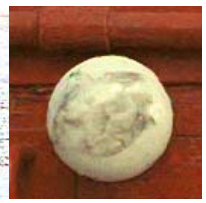

e) NLM

f) NLM error

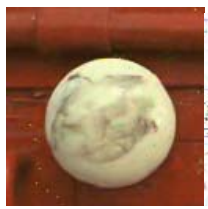

g) WESNR

h) WESNR error i)FMN-3DWTC. j) FMN-3DWTC Error.

Fig. 4. Filtered and Inverted error images (amplified by 5) for the image pic002 applying Bilateral, NLM, WESNR and FMN3DWT-C techniques for a mixture of noises: additive $(\sigma=$ $10)$ and random impulsive $(\%=10)$.

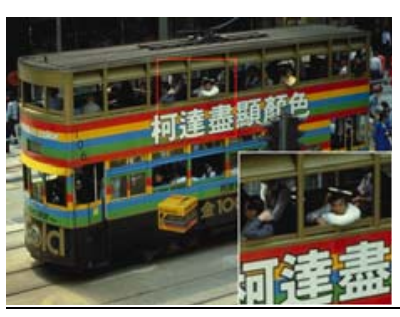

a) Original

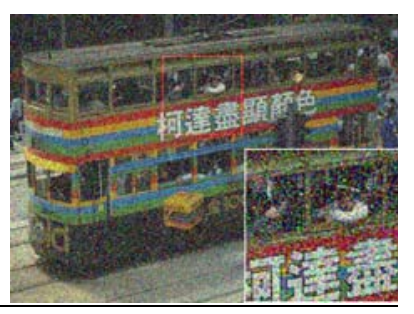

b) Noisy

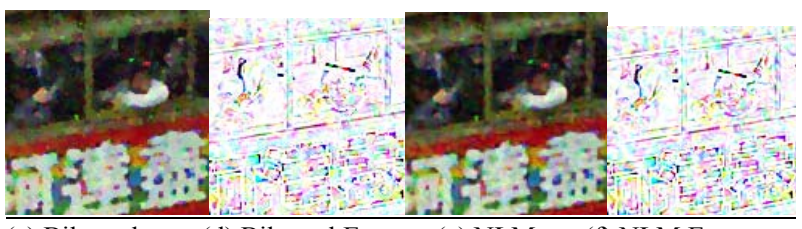

(c) Bilateral.

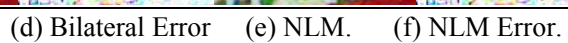

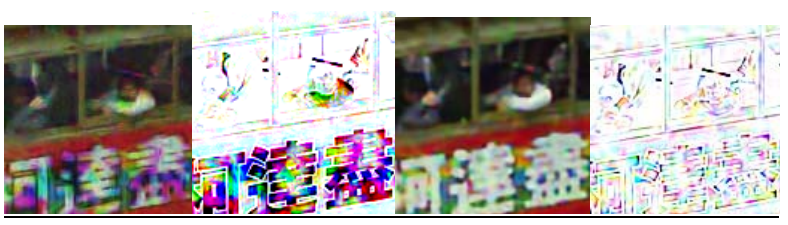

(g) WESNR. (h) WESNR Error (i) FMN-3DWTC (j) FMN-3DWTC

Error

Fig. 5. Filtered and Inverted error images (amplified by 3) for the image pic029 applying Bilateral, NLM, WESNR and FMN3DWT-C techniques for a mixture of noises: additive $(\sigma=$ $30)$ and random impulsive $(\%=30)$. 


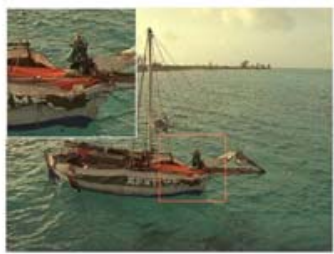

(a) Original image.

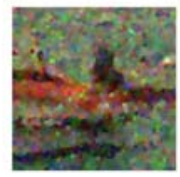

(c) Bilateral.

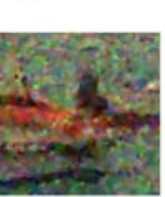

(g) NLM. ror

(h) NLM Error.

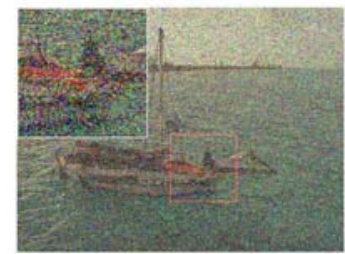

(b) Noisy image.

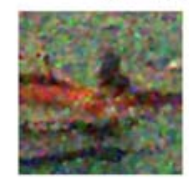

(e) Wiener.
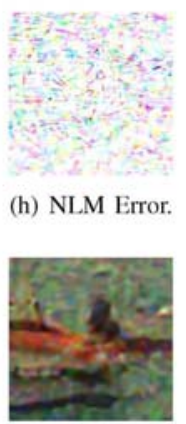

(k) FMN-3DWT- (1) FMN-3DWT-

C.

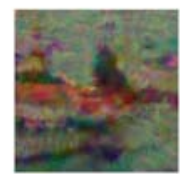

(i) WESNR.

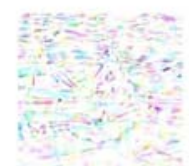

C Error.

Fig.6. Filetered and Inverted error images (amplified by 2) for the image pic059 applying Bilateral, Wiener, NLM, WESNR and FMN-3DWT in mixture of noises: additive $(\sigma=40)$ and random impulsive $(\%=40)$.

The values for PSNR and SSIM criteria in case of using different filters for images pic002, pic029, pic059, pic084 are shown in table 2 .

In case of multiplicative noise, the designed multiplicative denoising F-MNSR-DCT has demonstrated better performance in comparison with commonly used filtering techniques : Lee, Frost, Kuan and, anisotropic diffusion. Table 3 presents PSNR and SSIM values obtained in denoising of multiplicative distortions where novel filter appears demonstrate better performence.

Subjective visual perception results via analysis of filtered images and their error images for the state-of-the art filters and proposed F-MNSR-DCT are exposed in Fig. 7.

Table 2. The PSNR and SSIM values obtained for images pic002 (10; 10), pic029 (30; 30), pic059 (40; 40), and pic084 $(50 ; 50)$.

PSNR
\begin{tabular}{|l|l|l|l|l|l|}
\hline Image & $\begin{array}{l}\text { Bilater } \\
\text { al }\end{array}$ & $\begin{array}{l}\text { Wiene } \\
\text { r }\end{array}$ & NLM & $\begin{array}{l}\text { WESN } \\
\text { R }\end{array}$ & $\begin{array}{l}\text { FMN- } \\
\text { 3DW } \\
\text { T-C }\end{array}$ \\
\hline pic002 & 30.13 & 30.65 & 29.41 & 29.66 & $\mathbf{3 2 . 3 3}$ \\
\hline pic029 & 21.96 & 22.29 & 22.12 & 21.36 & $\mathbf{2 4 . 1 9}$ \\
\hline pic059 & 19.55 & 19.82 & 19.91 & 13.90 & $\mathbf{2 1 . 9 6}$ \\
\hline pic084 & 17.10 & 17.65 & 17.72 & 11.84 & $\mathbf{2 0 . 0 2}$ \\
\hline
\end{tabular}

SSIM
\begin{tabular}{|l|l|l|l|l|l|}
\hline Image & $\begin{array}{l}\text { Bilater } \\
\text { al }\end{array}$ & Wiener & NLM & $\begin{array}{l}\text { WES } \\
\text { NR }\end{array}$ & $\begin{array}{l}\text { FMN- } \\
\text { 3DW } \\
\text { T-C }\end{array}$ \\
\hline pic002 & 0.983 & 0.986 & 0.981 & 0.981 & $\mathbf{0 . 9 9 0}$ \\
\hline pic029 & 0.627 & 0.647 & 0.641 & 0.697 & $\mathbf{0 . 7 9 9}$ \\
\hline pic059 & 0.501 & 0.521 & 0.529 & 0.428 & $\mathbf{0 . 6 6 7}$ \\
\hline pic084 & 0.640 & 0.671 & 0.678 & 0.458 & $\mathbf{0 . 8 0 2}$ \\
\hline
\end{tabular}

Table 3. PSNR and SSIM values obtained in denoising of multiplicative distortions for filters: Lee, Frost, Kuan, Anisotropic diffusion and proposed filter F-MNSR-DCT.

\begin{tabular}{c|c|c|c|c|c|c|c|c|c|c}
\hline & \multicolumn{7}{|c|}{ PSNR } & \multicolumn{5}{c}{ SSIM } \\
\hline$\sigma$ & 10 & 20 & 30 & 40 & 50 & 10 & 20 & 30 & 40 & 50 \\
\hline Lee & 31.00 & 29.21 & 25.73 & 19.16 & 14.54 & 0.7308 & 0.7290 & 0.7254 & 0.7200 & 0.7132 \\
Froot & 32.93 & 29.94 & 25.86 & 19.09 & 14.55 & 0.8770 & 0.8559 & 0.8312 & 0.7982 & 0.7434 \\
Kuan & 29.76 & 28.32 & 25.27 & 19.02 & 14.48 & 0.8059 & 0.8098 & 0.7016 & 0.7833 & 0.7431 \\
Anisotrópica & 29.88 & 26.38 & 23.63 & 19.81 & 15.40 & 0.8088 & 0.8572 & 0.8393 & 0.7963 & 0.7617 \\
F-MNSR-DCT & 33.80 & 30.19 & 26.61 & 21.48 & 19.76 & 0.9043 & 0.8885 & 0.8414 & 0.8295 & 0.8006 \\
\hline
\end{tabular}

\section{Conclusion}

A novel filtering approach that has been employed in suppressing a mixture of additive-impulsive noises and in multiplicative denoising is presented. The experimental results demonstrate that our method exhibits better processing performance than state-of-theart techniques in suppressing mixed noise with varying texture characteristics and edges. Future work should be devoted to implementing the current filtering approach in video denoising.

Authors thank the Instituto Politecnico Nacional (Mexico) and Consejo Nacional de Ciencia y Tecnologia (Mexico) (grant 220347) for their support in realizing this work. 


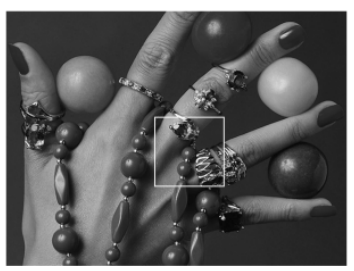

(a) pic015.

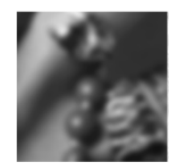

(e) Lee.

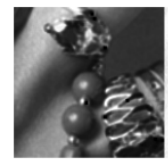

(i) Difusión anisótropica.

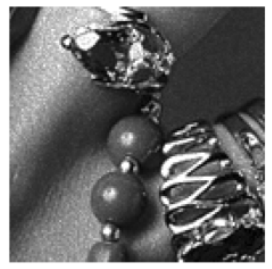

(b) $\operatorname{pic015}(\sigma=30)$.

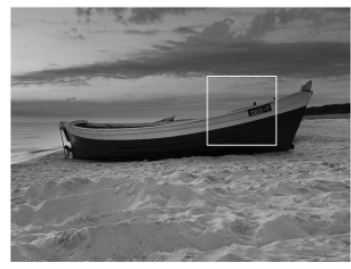

(c) pic091.

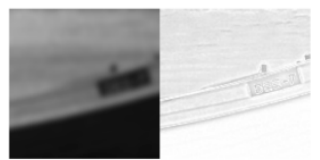

(g) Lee.

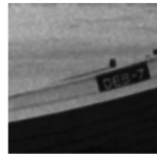

(k) Difusión anisótropica.

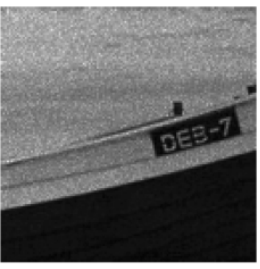

(d) $\operatorname{pic091}(\sigma=40)$.

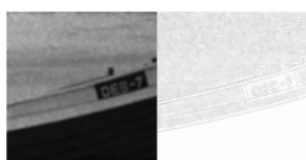

(h) Kuan.

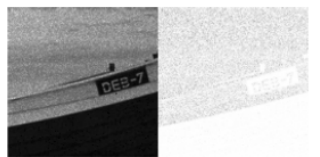

(l) F-MNSR-DCT.

Fig.7. Filtered and inverted error images for pic 015 and pic001 in case of multiplicative noise ( $\sigma=30, \sigma=40)$, respectively for different algorithms: Lee, Frost, Kuan, Anisotropic diffusion and proposed F-MNSR-DCT.

\section{References}

[1] Pitas, I. Digital image processing algorithms and applications. Wiley (2000).

[2] Young, I., Gerbrands, J., and Vliet, L. Fundamentals of image processing. TU Delft, Faculty of Applied Physics, Pattern Recognition Group (1995).

[3] Young, I. T.,. Gerbrands, J. J, and van Vliet, L. J. Fundamentals of Image Processing (v.2.3). Delft University of Technology (2007).

[4] Jain, P., and Tyagi, V. Information Sciences, 294(10). 164-181 (2015).

[5] Lukin, V., Fevralev, D., and Pogrebnyak, O. In: Proceedings Congress WAC. 1-6 (2012).

[6] Bahoura, M., and Ezzaidi H. Circuits, Systems, and Signal Processing, 31(3), 987-1015 (2012).

[7] Jin, Y., Jost, J., and Wang G. Journal of Mathematical Imaging and Vision, 48(1), 93-105 (2014).

[8] Buades, A., and Coll,, B. Computer Vision and Pattern, 2, 60-65 (2005).

[9] Dabov, K., Foi, Katkovnik, A. V., and Egiazarian, K. IEEE Trans. Image Proces. 16(8), 2080-2095 (2007).

[10] Palacios-Enriquez A., and Ponomaryov, V. In: IEEE Conference Proceedings. Conielecomp, 140-147 (2016).

[11] Melange, T., Nachtegael, M., Schulte, S., and Kerre, E. E. Image and Vision Comput., 29(6), 407-419 (2011). [12] Ponomaryov, V. I., Montenegro-Monroy, H., Gallegos-Funes, F., Pogrebnyak, O., and Sadovnychiy, S. Neurocomputing, 155, 225-246 (2015).
[13] Rosales-Silva, A. J., Gallegos-Funes, F. J., and Ponomaryov, V. I. Journal of Visual Communication and Image Representation. 23(1), 143-149 (2012).

[14] Lukac, R. Pattern Recognition Letters. 24(12), 1889-1899 (2003).

[15] Ng, P. E., and Ma, K. K. IEEE Trans. Image Proces. 15(6), 1506-1516 (2006).

[16] Xu G., and Tan, J. Circuits, Systems, and Signal Proces. 33(2), 421-435 (2014).

[17] Nasri, M., Saryazdi, S., and Nezamabadi-pour, H. Circuits, Systems, and Signal Proces. 32(4), 1839-1857 (2013).

[18] Veerakumar, T., K. Jagannath, R. P.. Subudhi, B. N.. and. Esakkirajan, S. Circuits, Systems, and Signal Proces.. 1-32 (2016).

[19] Zhang, Y., Tian, X., and Ren, P. Neurocomp. 140, 299-316 (2014).

[20]. Terol-Villalobos I. R., and Mendiola-Santiba nez, J. D. IET Image Proces. 8(3), 131-141 (2014).

[21] Jiang, J., Zhang, L., and Yang, J. IEEE Trans. Image Proces. 23(6), 2651-2662 (2014).

[22] Astola, J., Haavisto, P., and Neuvo, Y. Proc. IEEE, 78(4), 678-689 (1990).

[23] Bruckstein, A. M., Donoho, D. L., and Elad, M. SIAM Review, 51(1), 34-81 (2009).

[24] Lim, J. S. Two-dimensional signal and image processing. Prentice Hall (1990).

[25] Malinski L., and Smolka, B. Journal of Real-Time Image Proces. 1-22, (2016).

[26] Wang, Z., Bovik, A. C.,. Sheikh, H. R., Simoncelli, E. P. IEEE Trans. Image Proces. 13(4), 600-612 (2004). 